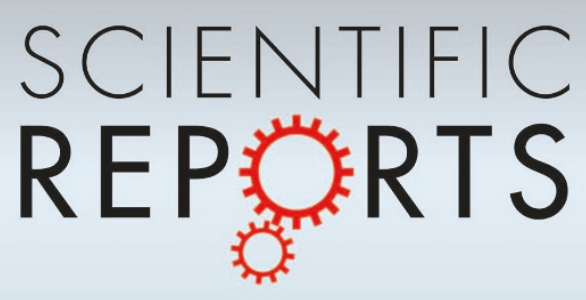

OPEN

SUBJECT AREAS:

OPTICAL TECHNIQUES

OPTICAL SENSORS

OPTOELECTRONIC DEVICES AND

COMPONENTS

TECHNIQUES AND

INSTRUMENTATION

Received

2 July 2013

Accepted

24 September 2013

Published

9 October 2013

Correspondence and requests for materials should be addressed to Y.T. (Tanyd@tsinghua. edu.cn)

\section{Response of microchip solid-state laser to external frequency-shifted feedback and its applications}

\author{
Yidong Tan', Shulian Zhang', Song Zhang', Yongqing Zhang² \& Ning Liv²
}

\begin{abstract}
${ }^{1}$ State Key Laboratory of Precision Measurement Technology and Instruments, Department of Precision Instruments, Tsinghua University, Beijing 100084, China, ${ }^{2}$ School of Mechanical Engineering, Nantong University, Nantong, Jiangsu 226019, China.
\end{abstract}

The response of the microchip solid-state Nd:YAG laser, which is subjected to external frequency-shifted feedback, is experimentally and theoretically analysed. The continuous weak response of the laser to the phase and amplitude of the feedback light is achieved by controlling the feedback power level, and this system can be used to achieve contact-free measurement of displacement, vibration, liquid evaporation and thermal expansion with nanometre accuracy in common room conditions without precise environmental control. Furthermore, a strong response, including chaotic harmonic and parametric oscillation, is observed, and the spectrum of this response, as examined by a frequency-stabilised Nd:YAG laser, indicates laser spectral linewidth broadening.

aser feedback, also called self-mixing interference, is a commonly observed phenomenon that can easily be caused by any surface in the light path of optical systems. Initially, laser feedback was regarded as a severely detrimental to laser performance, inducing instability in the laser's power and frequency ${ }^{1,2}$, as well as strong quantum noise $e^{3-5}$ and coherent collapse $e^{6-8}$. Thus, significant attention was placed on eliminating the external feedback. However, the fruitful phenomena in a laser diode subjected to external optical feedback attracted increasing interest, indicating the possibility of actively using laser feedback to improve laser performance to suit practical demands. Sacher and co-workers ${ }^{6}$ analysed the coherence collapse in a diode laser with optical feedback and were able to narrow the laser spectral linewidth. Utilising orthogonal polarisation optical feedback, $\mathrm{GHz}$ pulses of a single-mode diode laser were produced by $\mathrm{Cheng}^{9}$. In addition, polarisation switching and optical bistable states have been widely investigated ${ }^{10,11}$.

A common view about laser frequency-shifted feedback is that the laser resonator has gain amplification to the external feedback power. This amplification depends on the ratio of $\gamma_{c} / \gamma_{1}$, where $\gamma_{c}$ and $\gamma_{1}$, are, respectively, the damping rate of the laser cavity and the population inversion (i.e., the inverse of photon lifetime and of laser upper-level lifetime). For a laser diode, the typical value ${ }^{12}$ of this ratio is approximately $10^{3}$. Benefiting from this amplification of $\sim 10^{3}$, the weak feedback power reflected/scattered by a diffusing or absorbing surface of a measured target can be amplified by three orders of magnitude in a laser resonator, and thereby, detectable intensity modulation signals can be generated. From these intensity signals, the movement information of the measured target can be recovered. Thus, the gain amplification makes the laser diode sensitive to external feedback power and suitable for some cases of contact-free measurement (non-cooperative targets with diffusing or absorbing surfaces). For micro-chip solid-state lasers, this value ${ }^{12}$ of the ratio reaches as high as $\sim 10^{6}$. Consequently, these lasers can sense the extremely weak feedback power as low as $10^{-12}$, which permits broad application in fields such as tomography ${ }^{12,13}$, microscopy $y^{14}$, interferometry ${ }^{15}$, vibrometry ${ }^{16}$, and velocimetry ${ }^{17}$. However, it is not yet clear to what amount of feedback power, the laser can be used for heterodyne phase measurement. Additionally, this large amplification of $\sim 10^{6}$ will result in a strong response of the laser to the external feedback power even when that power is very weak. Some phenomena reported in diode lasers should also be observed in solid-state lasers, such as spectral linewidth broadening, chaotic spiking oscillation, and multiple stable states.

We have previously reported on the frequency characteristics of an Nd:YAG laser subjected to frequencyshifted feedback ${ }^{18}$. Only the frequency distribution in the low frequency region (i.e., in the frequency range around the laser's own relaxation oscillation frequency) was observed, and laser intensity information was not collected. In addition, the optical spectrum was not examined by frequency-stabilised lasers; thus, detailed spectrum information could not be obtained. Accordingly, it was difficult for us to judge whether the spectrum 
is broadened by the strong response of the laser to external feedback power. Moreover, the principle of a contact-free measurement scheme and its application were not performed.

In this report, we present the weak and strong response of the microchip solid-state Nd:YAG laser to external frequency-shifted optical feedback. For very weak feedback, the response of the laser to external frequency-shifted feedback exhibits continuous intensity modulation in amplitude and phase. From this intensity modulation, the movement information of a measured target can be extracted with high sensitivity, resolution and accuracy by heterodyne phase measurement. Based on this principle, a laser feedback interferometer is designed and developed to meet the demand of non-cooperative displacement/vibration measurement for all types of material surfaces, such as blackened aluminium, liquids, piezoelectric material, and even heated iron at high temperatures. In addition to weak response, with the increase of the feedback level, the laser displays a strong response to external feedback, which results in harmonic and parametric oscillations in the frequency domain and corresponding abrupt change in laser intensity and phase in the time domain. Through examination of a frequency-stabilised Nd:YAG laser, the spectrum is observed to be broadened.

\section{Results}

The experimental setup employed in this work is illustrated in Fig. 1. A $\phi 5 \mathrm{~mm} \times 1 \mathrm{~mm}$ Nd:YAG crystal microchip, with both surfaces coated to be highly reflective $(\mathrm{R} 1=99.8 \%, \mathrm{R} 2=99 \%)$ at the lasing wavelength of $1.064 \mu \mathrm{m}$, was employed to form a plane-parallel Fabry-Perot resonator. A fibre-coupled single-mode laser diode (not shown in Fig. 1) with a narrow linewidth $(<0.1 \mathrm{~nm})$ served as pump source, whose output was focused on the Nd:YAG crystal using a GRIN lens. The pump end of the Nd:YAG crystal was coated to be highly transmissive at the pump wavelength of $808 \mathrm{~nm}$. The lasing threshold was $\mathrm{P}_{\text {th }}=10 \mathrm{~mW}$. During the entire experiments, this microchip Nd:YAG laser worked in a linearly polarised $\mathrm{TEM}_{00}$ transverse mode. The path between the microchip lasers and the external feedback mirror $M$ is the so-called external feedback cavity or external cavity. A variable attenuator ATT was inserted into the external cavity to modify the feedback level. Here, the feedback level $\kappa$ is defined as the ratio of the feedback power to the laser output power.

The laser output is divided into two parts by the beam splitter $\mathrm{BS}_{1}$. The transmissive one is frequency-shifted by a pair of acousto-optic modulators $\left(\mathrm{AOM}_{1}\right.$ and $\mathrm{AOM}_{2}$, the central frequencies are $\Omega_{1}=$ $70.04 \mathrm{MHz}$ and $\Omega_{2}=70.08 \mathrm{MHz}$, respectively.). By carefully aligning the AOMs and external feedback mirror M, we shift the optical carrier frequency by an amount of $\Omega=2 \times(70.08-70.04)=$ $80 \mathrm{kHz}$ after a roundtrip of the laser beam.

The reflected beam is again separated into two parts by the beam splitter $\mathrm{BS}_{3}$. One part is detected by a photoreceiver (New Focus Model 1592, $3.5 \mathrm{GHz}$ ) accompanied by an oscilloscope to capture the laser intensity modulation and its power spectrum. The other part is combined with a frequency-stabilised Nd:YAG laser (Innolight M500, linewidth $100 \mathrm{kHz}$ ). The beat signal is sent to another photoreceiver (New Focus Model 1592, 3.5 GHz) followed by a spectrum analyser (Agilent N9020B) to capture the spectrum distribution of the beat signal.

Actually, after a roundtrip in the external cavity, four beams can return to the laser resonator, whose frequency shifts are $\Omega, \Omega / 2, \Omega_{1}$, and $\Omega_{2}$ for each beam. Owing to the limited bandwidth of the relaxation oscillation ${ }^{15}$, the microchip laser cannot respond to feedback light whose frequency shift is much larger than the relaxation oscillation frequency $\mathrm{f}_{\mathrm{R}}=290 \mathrm{kHz}$ at the pump level of $\mathrm{P} / \mathrm{P}_{\text {th }}=2$. Consequently, the two beams of frequency shifts $\Omega_{1}$, and $\Omega_{2}$ can be omitted. In Fig. 1, the red line with the heavy arrow represents the feedback light with the frequency shift at $\Omega$, and the blue line with light arrow represents the feedback light with the frequency shift at $\Omega / 2$.

Weak response to external frequency-shifted feedback. By setting the very weak feedback level ( $\kappa$ is approximately $10^{-6}$ ), the microchip Nd:YAG laser exhibits weak response to external feedback, as illustrated in Fig. 2 (a). The Fig. 2 (a1) is the laser intensity, and Fig. 2 (a2) is the corresponding power spectrum. $I_{f}$ represents the laser intensity with feedback, and $\mathrm{I}_{0}$ represents the laser intensity without feedback. The laser intensity is sinusoidally modulated at a frequency of $\Omega=80 \mathrm{kHz}$, which is the shifted frequency of the light after one roundtrip propagation in the external cavity. The insert shows a temporal zoom of the laser intensity. The corresponding power spectrum (observed by the fast Fourier transform function of the oscilloscope) displays two oscillating frequencies, i.e., $\Omega=$ $80 \mathrm{kHz}$, and $\mathrm{f}_{\mathrm{R}}=290 \mathrm{kHz}$. The signal envelope is approximately $5 \mathrm{kHz}$ corresponds to the noise of the photoreceiver.

By slightly increasing the feedback level a little ( $\kappa$ is approximately $\left.2 \times 10^{-6}\right)$, an expected intensity increment occurs, which is the intensity modulation depth in Fig. 2 (b1) and is twice that in Fig. 2 (a1). Although the power spectrum exhibits slight harmonic oscillations at $2 \Omega, 3 \Omega$ and so on, the laser intensity remains sinusoidally modulated. The results are quite different from the results in Fig. 9, where the strong feedback causes large resonant quantum noise of $-70 \mathrm{~dB}$ and the multiple solutions to laser frequency. Consequently, the laser frequency skips the unstable region of multiple solutions

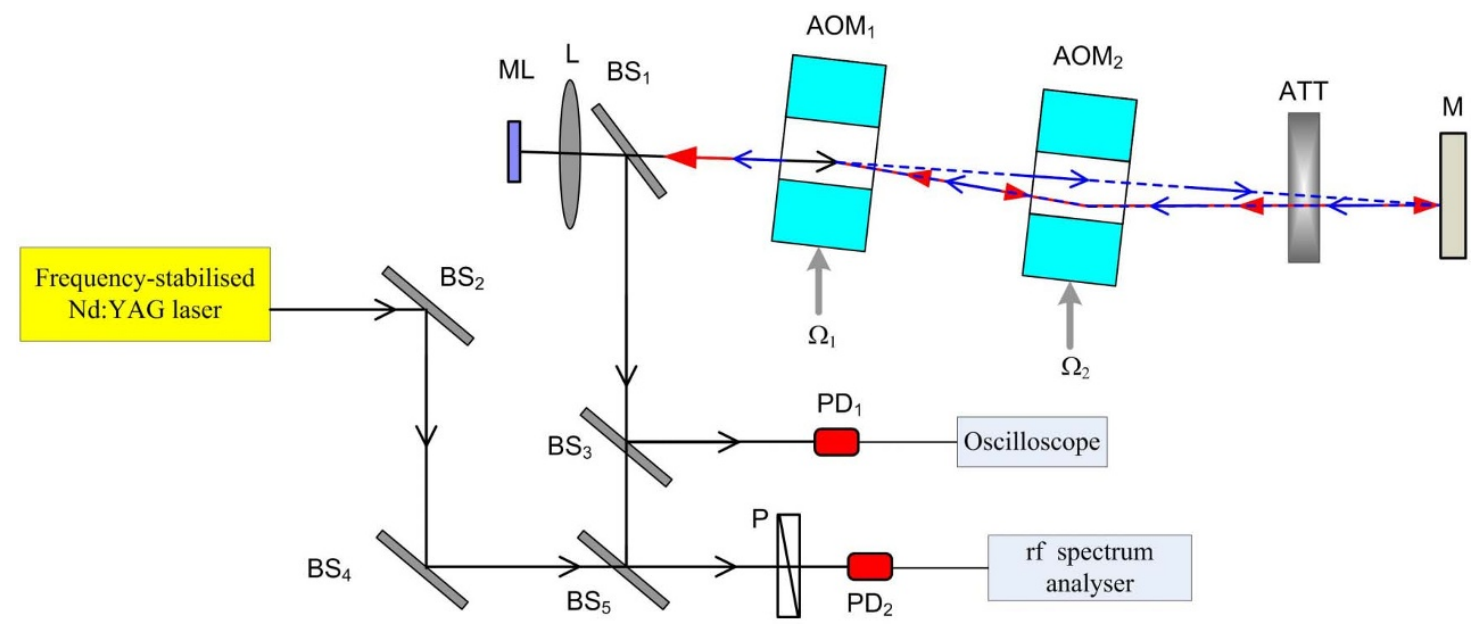

Figure 1 | Schematic of experimental setup. ML: microchip solid-state Nd:YAG laser crystal, L: lens, BS ${ }_{1-4}$ : beam splitter, AOM 1,2 : acousto-optic modulator, ATT: attenuator, $\mathrm{M}$ : feedback mirror (or target to be measured), $\mathrm{PD}_{1,2}$ : photoreceiver, and P: polariser. 

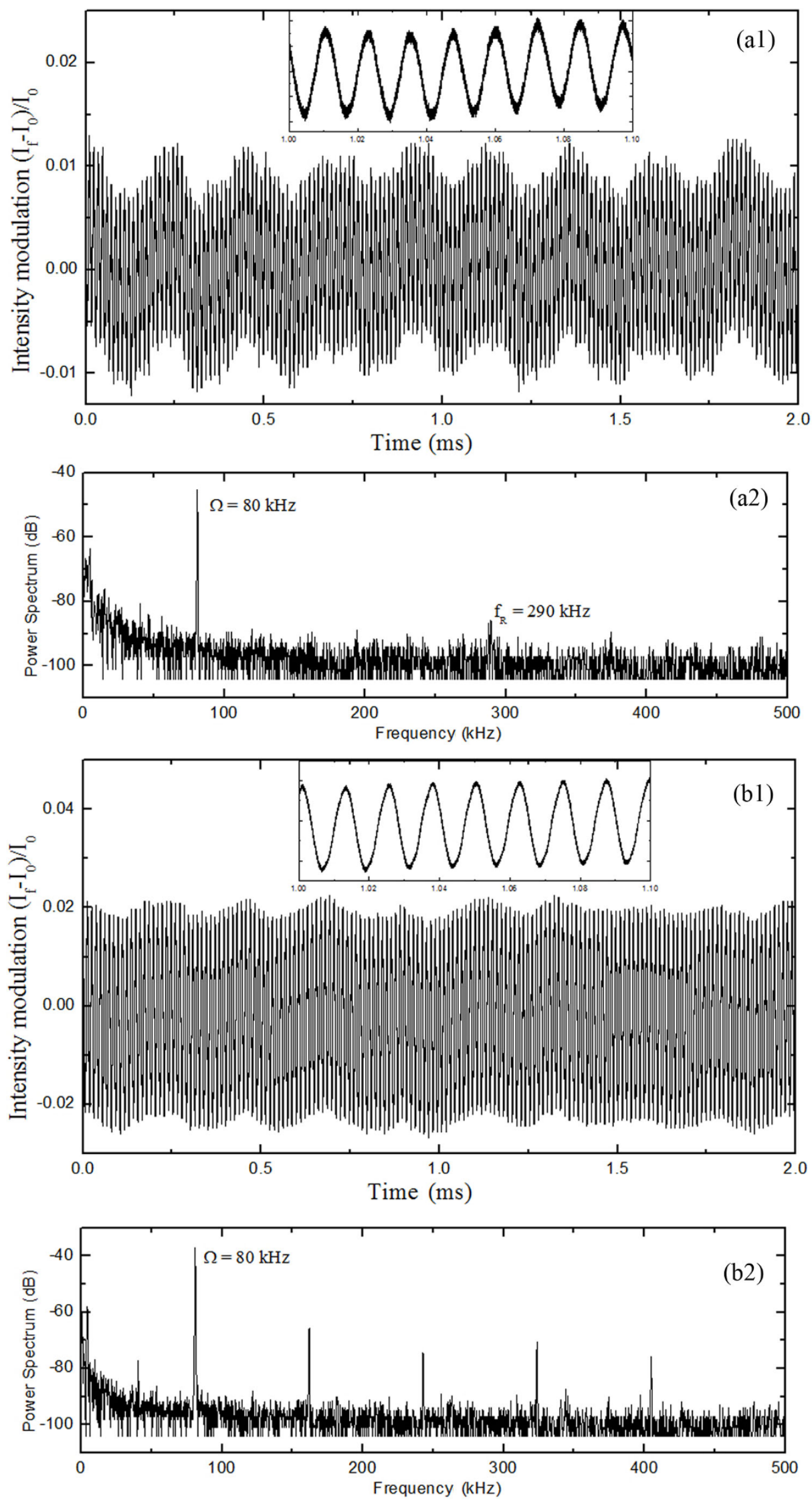

Figure $2 \mid$ Weak response of the laser to external frequency-shifted feedback: (a) $\kappa=10^{-6}$ and (b) $\kappa=2 \times 10^{-6}$. The insert displays a temporal zoom of the intensity. 
and jumps down to the neighboring stable region. For the weak level, there is only one solution, and the laser is continuously modulated by external feedback. We will explain this phenomenon in detail in the discussion section. Here, the intensity modulation depth proportionally increases with that of the feedback level $\kappa$, which's why this response is called weak (linear) response.

When the laser is subjected to external frequency-shifted feedback with a weak level $\left(\kappa<10^{-4}\right)$, the laser output exhibits a weak (linear) response to the feedback field, whose intensity amplitude linearly increases with the feedback level and the phase of the light is continuously modulated without abrupt change. If the feedback mirror $M$ in Fig. 1 is replaced by a moving target, its movement information will be loaded on a continuous phase change in the $\Omega$-modulated laser intensity. Using a heterodyne phase measurement, we can obtain the movement information of the target. In addition, the weak response is validated under a weak feedback level $\left(\kappa<10^{-4}\right)$, which makes it appropriate for the contact-free measurement of materials with low or even extremely low reflectivity.

Based on this character, a scheme of non-cooperative measurement is developed to achieve high resolution, high sensitivity, and environmental robustness in the later sections.

Contact-free measurement scheme with high accuracy, sensitivity and environmental robustness. Based on the weak response of the laser subjected to the weak feedback level, a scheme of contact-free measurement is developed to achieve high accuracy, high sensitivity and environmental robustness, as illustrated in Fig. 3.

Except for the reference mirror $\mathrm{M}_{\mathrm{r}}$, most of the components used in Fig. 3 are the same as those presented in Fig. 1. The laser beam (frequency: $\omega$ ) in turn passes through two AOMs. Then, after $\mathrm{AOM}_{2}$, one of the resultant beams (frequency: $\omega+\Omega / 2$ ), denoted by the red line with the heavy arrow in Fig. 3, serves as the probing beam and impinges on the measured target $(\mathrm{M})$. The reflection by the target then returns to the microchip laser resonator along its incoming path, forming the feedback measurement arm (frequency: $\omega+\Omega$ ). The other part, denoted by the blue line with light arrow, remains $\omega$ because it is not diffracted. This part is reflected by the $M_{r}$ back to the resonator along the path of the measurement arm, forming the feedback reference arm (frequency: $\omega+\Omega / 2$ ). Thus, the total path of the measurement and reference arms $\mathrm{L}_{\mathrm{m}}$ and $\mathrm{L}_{\mathrm{r}}$ can be written as

$$
\begin{gathered}
\mathrm{L}_{m}=2 \times\left(\mathrm{L}_{0}+\mathrm{L}_{\mathrm{d} 1}+\mathrm{D}_{0}+\Delta \mathrm{L}\right) \\
\mathrm{L}_{r}=2 \times \mathrm{L}_{0}+\mathrm{L}_{\mathrm{d} 0}+\mathrm{L}_{\mathrm{d} 1}
\end{gathered}
$$

where $L_{0}$ is the light path between the laser and the $A O M_{1} ; L_{d 1}$ is the light path of the diffracted beam between $A O M_{1}$ and $M_{r} ; L_{d 0}$ is the light path of the un-diffracted beam between $A_{O} M_{1}$ and $M_{r} ; D_{0}$ is the light path between $\mathrm{M}_{\mathrm{r}}$ and the initial position of $\mathrm{M}$; and $\Delta \mathrm{L}$ is the displacement to be measured.

Therefore, the optical path changes in the arms of measurement and reference are obtained

$$
\begin{gathered}
\Delta \mathrm{L}_{m}=2 \times\left(\Delta \mathrm{L}_{0}+\Delta \mathrm{L}_{\mathrm{d} 1}+\Delta \mathrm{D}_{0}+\Delta \mathrm{L}\right) \\
\Delta \mathrm{L}_{r}=2 \times \Delta \mathrm{L}_{0}+\Delta \mathrm{L}_{\mathrm{d} 0}+\Delta \mathrm{L}_{\mathrm{d} 1}
\end{gathered}
$$

Because it is close enough for the arms of measurement and reference to be subjected to the same environmental disturbance, the optical path changes induced by the environment can be assumed to be the same, i.e., $\Delta \mathrm{L}_{\mathrm{d} 1} \approx \Delta \mathrm{L}_{\mathrm{d} 0}$.

Then, we can obtain

$$
\Delta \mathrm{L}_{m}=\Delta \mathrm{L}_{r}+2 \Delta \mathrm{D}_{0}+2 \Delta \mathrm{L}
$$

Thus,the corresponding phase change can be written as

$$
\Delta \phi_{\mathrm{m}}=\Delta \phi_{r}+\Delta \phi_{D 0}+2 \mathrm{k} \Delta \mathrm{L}
$$

where $k$ is the wave vector of the laser beam. In the measurement, the reference mirror is placed close enough to the initial position of the measured target. Therefore, the phase change $\Delta \phi_{\mathrm{D} 0}$ in this path $\left(\Delta \mathrm{D}_{0}\right.$ $\approx 0$ ) can be omitted. Then, we obtain

$$
\Delta \phi_{\mathrm{m}}=\Delta \phi_{r}+2 \mathrm{k} \Delta \mathrm{L}
$$

This equation clearly indicates that the phase change in the measurement arm contains two parts: one is the real displacement to be measured, and the other is the phase change in the reference arm, which represents the environmental disturbance. Consequently, the difference between the measurement and reference arms really reflects the movement of the measured target. This scheme compensates for the environmental disturbance between the laser and reference mirror $M_{r}$. Thus, in the practical use, it is important to place the reference mirror $\mathrm{M}_{\mathrm{r}}$ close enough to the measured target.

To obtain the real displacement of the measured target, the phase changes in the measurement and reference arms in Eq. (7) should be recovered from the intensity modulation signals of the laser frequency-shifted feedback system.

For weak feedback, when both the frequency-shifted lights at $\Omega$ and $\Omega / 2$ are reflected/scattered back into the laser resonator, the laser output is modulated at two frequencies separately as follows:

$$
\begin{gathered}
\frac{\Delta I_{m}}{I_{0}}=\sqrt{\kappa} G(2 \Omega) \cos \left(2 \pi \Omega t+\phi_{m}+\varphi_{0 m}\right) \\
\frac{\Delta I_{r}}{I_{0}}=\sqrt{\kappa} G(\Omega) \cos \left(\pi \Omega t+\phi_{r}+\varphi_{0 r}\right)
\end{gathered}
$$

where $\Delta \mathrm{I}$ is the laser intensity modulation; $\kappa$ is the feedback level; $\mathrm{G}(\Omega)$ is a frequency-dependent gain amplification factor ${ }^{12,15,16,17,18}$ that reaches its maximum $\gamma_{\mathrm{c}} / \gamma_{1}$ when $\Omega$ approaches $\mathrm{f}_{\mathrm{R}} ; \Omega=$ $80 \mathrm{kHz}$ is the frequency shifting; $\phi$ is the phase of the light carrying the information of the measured targets; and $\varphi_{0}$ is the fixed initial phase. The subscript symbols $m$ and $r$ represent the measurement and reference arms, respectively.

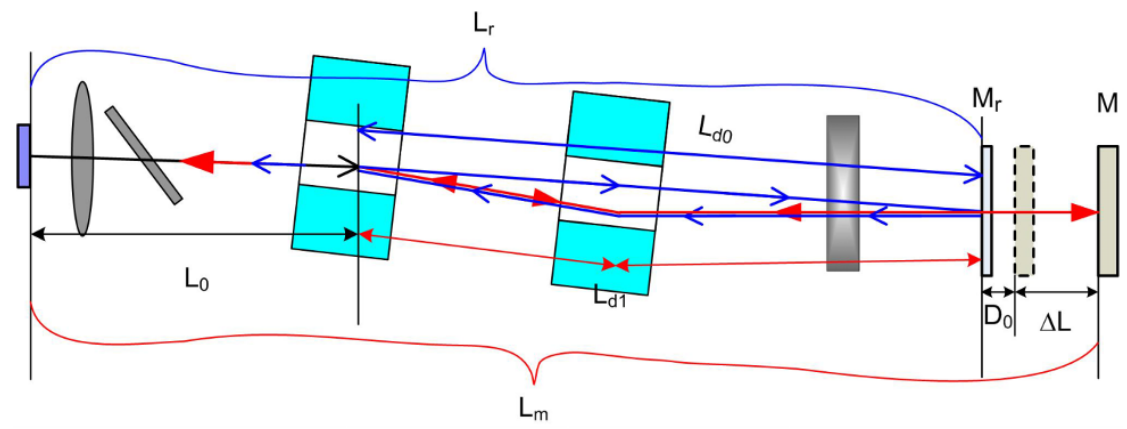

Figure 3 Schematic of contact-free measurement with quasi-common path compensation. 


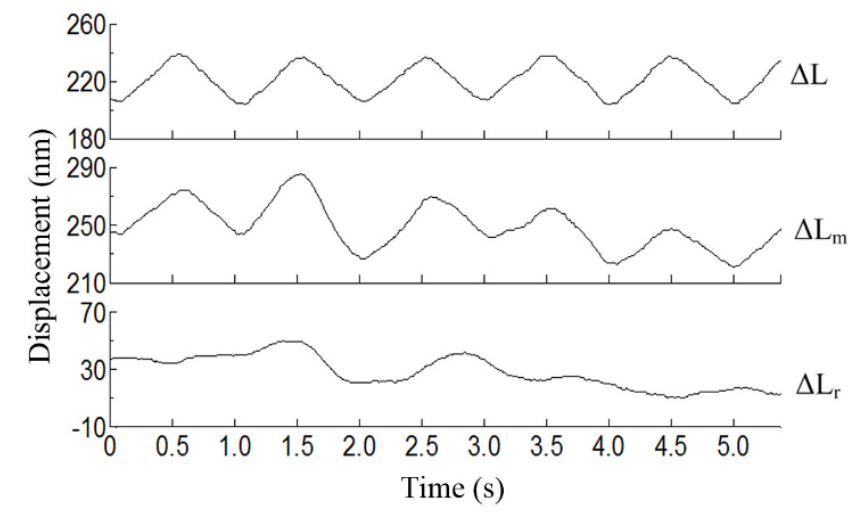

Figure 4 | Contact-free measurement of servo-controlled PZT stage under common room conditions.

Compared with traditional interference, the intensity modulation has an extra gain factor $G(\Omega)$, whose value ranges from $10^{4}$ to $10^{6}$. Therefore, even if the reflected/scattered light is extremely weak such as $10^{-12}$, the relative intensity modulation amplitude can reach $100 \%$, which implies it has high sensitivity to external feedback. Thus, this characteristic promises the ability of contact-free measurement.

Using a heterodyne phase measurement technique, the phase changes $\Delta \phi_{\mathrm{m}}$ and $\Delta \phi_{\mathrm{r}}$ in Eq. (8) and (9) can be demodulated, respectively. Then, according to Eq.(7), their difference $\left(\Delta \phi_{\mathrm{m}}-\Delta \phi_{\mathrm{r}}=2 k \times\right.$ $\Delta \mathrm{L})$ accurately reflects the real movement or displacement $(\Delta \mathrm{L})$ of the targets with an accuracy on the nanometer level under common room condition without precise temperature control and vibration isolation.

To verify the points discussed above, the scheme is used to directly measure the displacement (movement) of a servo-controlled PZT stage (PI Model P-762) driven by a triangular waveform with an amplitude of approximately $40 \mathrm{~nm}$. The laser beam impinges on the stage's surface, and the reflected/scattered light returns to produce a $\Omega$-frequency modulated laser intensity. By demodulating the signals from the measurement and reference arms, the displacement (optical path change) is obtained and is shown in Fig. 4. The $\Delta \mathrm{L}_{\mathrm{r}}$ results indicate that the light path change in the reference arm induced by the environmental disturbance is approximately $40 \mathrm{~nm}$, which is at the same level as the stage's movement amplitude $\Delta \mathrm{L}$. Consequently, it is difficult to judge the stage's displacement from the $\Delta \mathrm{L}_{\mathrm{m}}$, which is submerged into the environmental disturbance $\Delta \mathrm{L}_{\mathrm{r}}$. After the compensation of subtracting $\Delta \mathrm{L}_{\mathrm{r}}$ from $\Delta \mathrm{L}_{\mathrm{m}}, \Delta \mathrm{L}$ accurately reflects the movement of the stage with an amplitude of $40 \mathrm{~nm}$. It is concluded that the contact-free measurement with nanometre accuracy and environmental robustness can be achieved by a combination of frequency multiplexing (environmental compensation) and frequency-shifted feedback (gain amplification).

Based on this principle, a compact laser feedback interferometer was developed as shown in Fig. 5. To simultaneously demodulate the measurement and reference signals, a commercial phase meter is used, whose effective resolution is about $0.1^{\circ}$, corresponding to $0.14 \mathrm{~nm}$ resolution of displacement. Given the influence of the residual dead path $\left(\mathrm{D}_{0}\right.$ in Fig. 3$)$ on the measurement, the effective resolution of displacement measurement is evaluated as about $1 \mathrm{~nm}$. During the measurement process, the laser beam emitted from the instrument output directly impinges onto the surface of the targets to be measured, and then the feedback light generates intensity modulation, from which information about the targets can be recovered. Note that an attenuator should be added to decrease the feedback power if the reflected or scattered light is too strong. In remote sensing where the target is several metres far from the instrument, to guarantee high accuracy, a movable reference mirror $M_{r}$ is placed outside but near the measured target. Thus, the large dead path between the target and the instrument can be compensated for. Of course, the movable reference mirror $M_{r}$ is a sophisticated arrangement of mirrors and lens to ensure the feedback reference arm can return to the resonator parallel to the measurement arm.

Application in vibration measurement. The hysteretic characteristic of the piezoelectric ceramics supplied by PI Company was directly measured using the instrument above. The results are presented in Fig. 6 (left). We also tested the vibration of the piezoelectric ceramics at different frequencies ranging from $1 \mathrm{~Hz}-10 \mathrm{kHz}$ with different amplitudes from several tens to hundreds of nanometres. A typical result for vibration test at a frequency of $6 \mathrm{kHz}$ with an amplitude of tens of nanometres is presented in Fig. 6 (right). The piezoelectric thin films were also successfully measured using this instrument with an amplitude of tens of nanometres at common room conditions.

Application in liquid evaporation. The common approaches ${ }^{19-21}$ for measuring the liquid evaporation involve capacitive sensors, a fibre liquid level sensor, or laser triangulation. The first two approaches are not convenient because of the contact measurement. The last approach requires frequent calibration based on the measured targets.

We used the instrument presented above to measure the evaporation rates of four types of liquids: (1) water, (2) alcohol, (3) acetone, (4) ether. The measurement laser beam is turned $90^{\circ}$ down onto the surface of the liquid directly. The fall of the liquid surface is transformed into the optical path change in the measurement arm and thus, it is detected.

The experimental results illustrated in Fig. 7 reveal that after 20 minutes, the levels of the liquids decline as a result of evaporation as follows: water, $41.165 \mu \mathrm{m}$; alcohol, $206.098 \mu \mathrm{m}$; acetone, 1117.854 $\mu \mathrm{m}$; and ether, $2818.231 \mu \mathrm{m}$. The evaporation rate is expressed by the declined height divided by the time. Therefore, the evaporation rates for these four liquids are as follows: water, $34 \mathrm{~nm} / \mathrm{s}$; alcohol, $172 \mathrm{~nm} / \mathrm{s}$; acetone, $932 \mathrm{~nm} / \mathrm{s}$; and ether, $2349 \mathrm{~nm} / \mathrm{s}$.

Other experiments indicate that the evaporation rate is higher in the afternoon than in the morning due to the increasing temperature. The evaporation rate decreases when the liquid is maintained in a closed container due to vapor saturation.

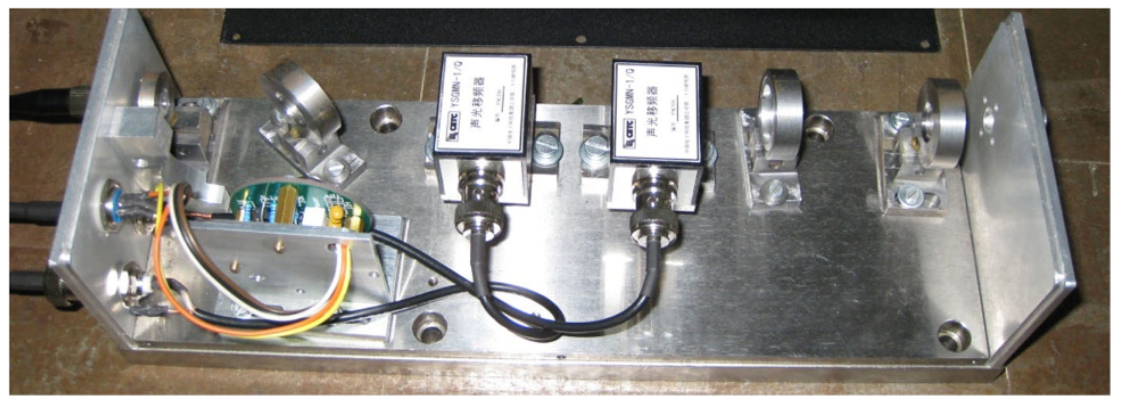

Figure 5 | Laser feedback interferometer based on external frequency-shifted feedback. 

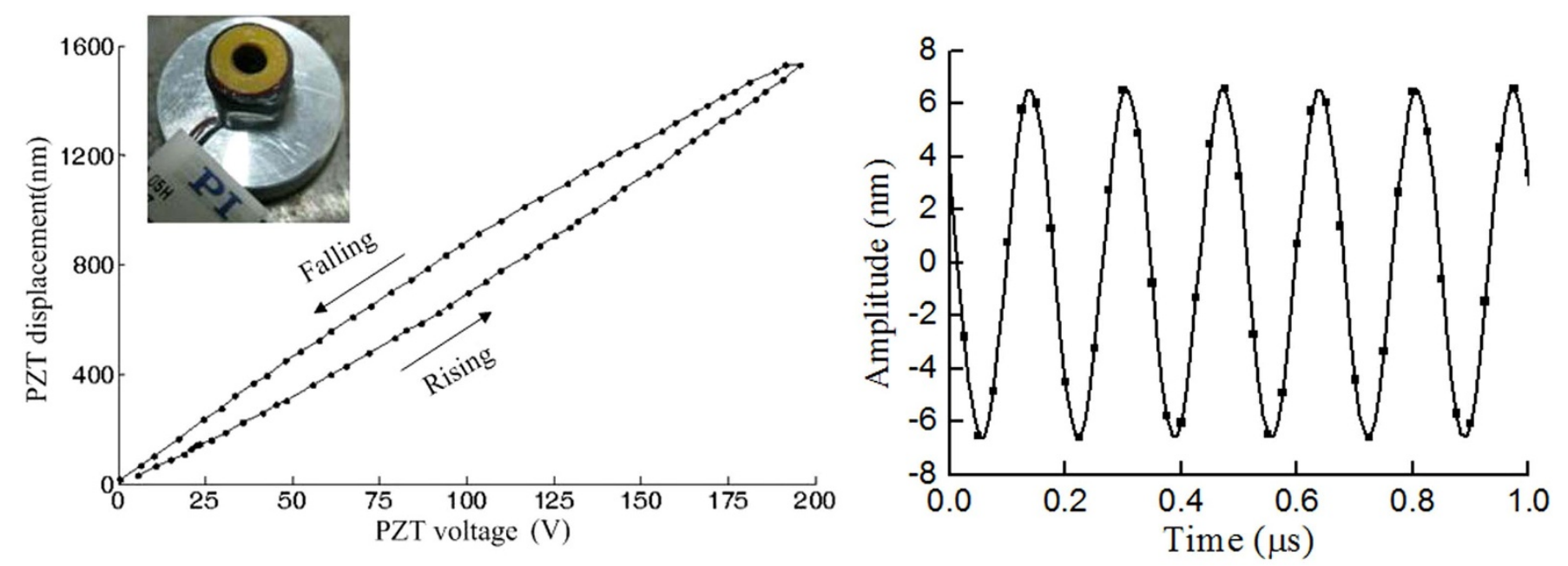

Figure 6 | Contact-free measurement for the hysteresis loop and vibration of piezoelectric ceramics: hysteresis loop (left) and vibration (right).

Application in thermal expansion of materials at high temperature. Previous methods ${ }^{22,23}$ for thermal expansion measurement include optical interference, X-ray, optical and mechanical lever, and density measurements; most of these methods require preprocessing of the surface, a certain geometric shape of the materials, or need cooperative reflective mirrors.

The laser feedback interferometer is expected to solve the above problem. First, 45\# steel is heated in a muffle furnace, whose temperature can be set from room temperature (approximately $20^{\circ} \mathrm{C}$ ) to $1200^{\circ} \mathrm{C}$. In the front surface of the muffle furnace, a hole is drilled to pass the measurement light. In the experiments, the beam emitted from the laser feedback interferometer passes through the hole into the muffle furnace and impinges on the surface of the $45 \#$ steel. Then, the reflected or scattered light returns back into the laser resonator to generate the $\Omega$-frequency modulation in laser intensity, from which the thermal expansion (optical path change) of the steel at different temperatures can be determined.

The change in the optical path (i.e., the displacement between the instrument and the surface of the steel) is used to represent the thermal expansion of the steel. The results measured here can qualitatively reflect the thermal expansion of the steel to some degree.

As shown in Fig. 8, the entire heating process lasts approximately 30 minutes, and the optical path change (displacement) between the instrument and the steel is approximately 150 microns. The insert on the left is the initial state of the 45\# steels (not the same one but the same material), and the insert on the right shows the final state of the steels heated. Clearly, its property has changed as a result of the

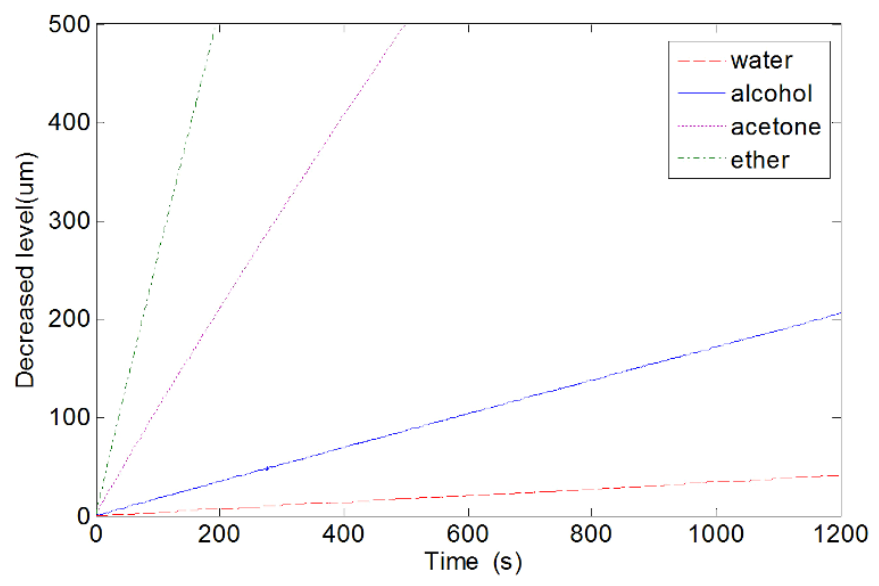

Figure 7 | Liquid evaporation measuremnt for serveral types of liquids. chemical reaction. In the experiments, the temperature in the muffle furnace is changed from $20^{\circ} \mathrm{C}$ to $500^{\circ} \mathrm{C}$. The reflectivity of the steel surface decreases with increasing temperature due to strong oxidation. Above $500^{\circ} \mathrm{C}$, the reflectivity of the steel is extremely low that the feedback signal cannot be adequately detected using the present scheme. A lock-in amplifier could possibly be used to further improve the detectability.

Strong response to external frequency-shifted feedback. When the amount of feedback is increased to $10^{-3}$ or even higher, a strong response clearly results, as demonstrated in Fig. 9. Both the feedback light frequency shifted at $\Omega$ and $\Omega / 2$ returns to the laser resonator, shown in Fig. 9 (a). Therefore, the intensity is modulated by these two frequencies. Unlike the cases in Fig. 2, the spiking pulses superpose on the weak response signals. Every spiking pulse represents an abrupt change in laser frequency caused by strong frequency-shifted feedback. The power spectrum exhibits strong harmonic $(2 \Omega, 3 \Omega, 4 \Omega \ldots)$ and parametric $(\Omega / 2+\Omega, \Omega / 2+2 \Omega$, $\Omega / 2+3 \Omega$...) oscillations resulting from these two frequencies. Additionally, the parametric oscillation frequency $(280 \mathrm{kHz})$ resonates with the relaxation oscillation frequency $\left(f_{R}=290 \mathrm{kHz}\right)$. Consequently, compared with Ref18, the parametric oscillation peaks $\left(f_{R}+\Omega / 2, f_{R}-\Omega / 2, f_{R}+\Omega, \ldots\right)$ related to the relaxation oscillation frequency vanish. Only the harmonic peaks at $\Omega$ and the mixed parametric peaks between $\Omega$ and $\Omega / 2$ survive. Simultaneously, the laser quantum noise $(-80 \mathrm{~dB})$ is stronger than that in Fig. $2(-100 \mathrm{~dB})$. The insert indicates that the amplitude and phase of the laser intensity break abruptly, where the frequency of the laser is thought to also jump sharply. This result is similar to the cases occurring in diode lasers ${ }^{25}$. The laser frequency is modulated by the phase of the external feedback light. At the strong feedback level, the modulation of the external feedback is so large that multiple solutions of laser frequency co-exist in the laser feedback system ${ }^{25,26}$. However among these solutions, some are unstable and violate the stability criteria ${ }^{26}$. Consequently, during the modulation, when the laser frequency is tuned to the region of unstable solutions, the frequency suddenly jumps to the stable one in the nearest neighbor period. A detailed interpretation will be provided in the discussion section. In this feedback regime, the intensity modulation depth no longer increases with the feedback level $\kappa\left(>10^{-3}\right)$, which is accordingly called the strong (nonlinear) response to external feedback. However, a more complicated spectrum distribution occurs.

For strong feedback level, a sharp change such as a spiking pulse in the laser intensity indicates that it is not suitable for heterodyne phase measurement. Although electronic filter can be used to filter the irregular spiking pulses, this filter can cause an extra phase change 


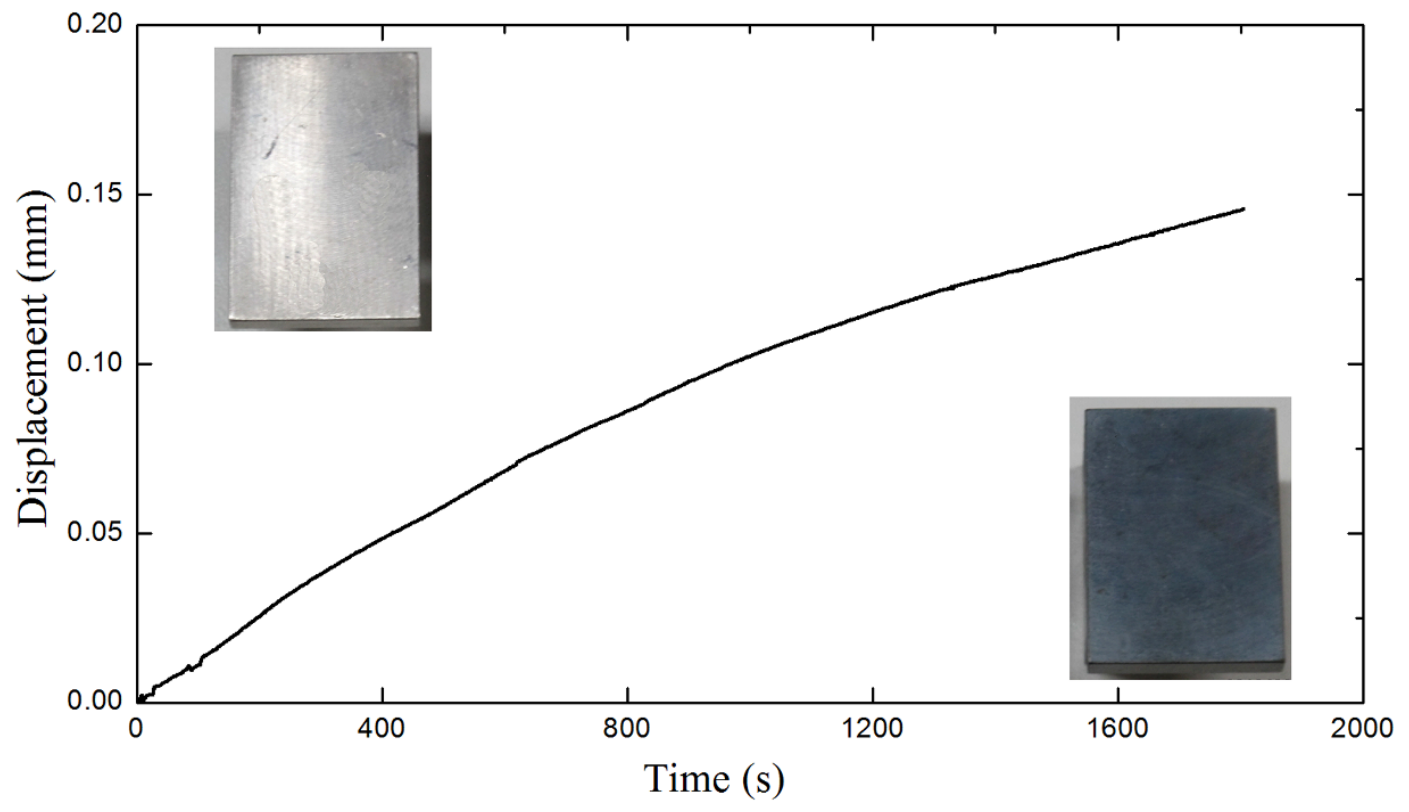

Figure 8 Thermal expansion of 45 \# steel with temperature change from $20^{\circ} \mathrm{C}-500^{\circ} \mathrm{C}$.

in the measurement signals, which causes the considerable errors in measurements on the nanometre level. However, the fruitful information of the power spectrum, as shown in Fig. 9 implies that the frequency of the laser subjected to the external frequency-shifted strong feedback is greatly affected. Consequently, in the following section, the optical spectrum distribution is examined using a frequency-stabilised Nd:YAG laser (Innolight M500, linewidth $100 \mathrm{kHz})$.

Spectrum broadening induced by strong response. To observe the optical spectrum, the microchip Nd:YAG subjected to external frequency-shifted feedback is compared with a frequency-stabilised $\mathrm{Nd}$ :YAG laser, whose linewidth is approximately $100 \mathrm{kHz}$. When the feedback loop is off, the beat frequency is located near the frequency of $1 \mathrm{GHz}$ around, whose linewidth is approximately $3 \mathrm{MHz}$, as indicated in Fig. 10. Given that the linewidth of the frequencystabilised Nd:YAG laser is only $100 \mathrm{kHz}$, we can qualitatively determine that the linewidth of the microchip Nd:YAG laser is at most $3 \mathrm{MHz}$. When the feedback loop is on, the linewidth of the beat frequency is greatly broadened to about $30 \mathrm{MHz}$. Thus, the linewidth of the microchip laser is approximately $30 \mathrm{MHz}$ under strong external frequency-shifted feedback. Although it is called strong feedback, the feedback level is only $2 \times 10^{-3}$. If it is largely increased, the linewidth of the laser is expected to be further broadened to several hundreds of $\mathrm{MHz}$.

We also verify the case of weak feedback. The linewidth hardly broadens under this condition and, thus, does not affect the application of the frequency-shifted feedback on precision measurement.

\section{Discussion}

In the presence of frequency-shifted optical feedback, the dynamic behaviour of a re-injected laser can be described using the modified Lang-Kobayashi equation ${ }^{16-18}$ :

$$
\begin{aligned}
\frac{d N(t)}{d t} & =\gamma_{1}\left[N_{0}-N(t)\right]-B N(t) E(t)^{2} \\
\frac{d E(t)}{d t} & =\frac{1}{2}\left[B N(t)-\gamma_{c}\right] E(t) \\
& +\gamma_{c} \sqrt{\kappa} \cos [2 \pi(\Omega t-\omega \tau)] E(t)
\end{aligned}
$$

$$
\frac{d \Phi}{d t}=\omega_{c}-\omega+\frac{\alpha}{2} B N+\gamma_{c} \sin [2 \pi \Omega t-\omega \tau]
$$

where $N(t)$ is the population inversion, $E(t)$ is the amplitude of the laser electric field, $\Phi$ is the phase of the light, $\omega_{c}$ is the laser cavity frequency, $\omega$ is the optical running laser frequency, $\Omega$ is the frequency-shifting by the AOMs, and $\tau$ is the roundtrip time in the external cavity. $\mathrm{B}$ is the Einstein coefficient, $\gamma_{1} \mathrm{~N}_{0}$ is the pumping rate, $\gamma_{1}$ is the decay rate of the population inversion, $\gamma_{c}$ is the laser cavity decay rate, $\kappa$ is the effective laser feedback level, and $\alpha$ is the linewidth enhancement factor in solid-state microchip lasers.

Solving Eq.(10) above, the following equations are obtained:

$$
\begin{gathered}
\omega=\omega_{c}+\frac{\alpha}{2} \gamma_{c}-\frac{\alpha}{\sqrt{1+\alpha^{2}}} \gamma_{c} \sqrt{\kappa} \sin \left(2 \pi \Omega t-\omega \tau+\operatorname{tg}^{-1} \alpha\right) \\
\frac{\Delta I}{I_{0}}=\sqrt{\kappa} G \cos \left(2 \pi \Omega t-\omega \tau+\varphi_{0}\right)
\end{gathered}
$$

Similar to the cases reported for diode lasers ${ }^{24}$, this transcendental Eq. (11) has only one solution for $\omega$, when $\frac{\gamma_{c} \tau \sqrt{\kappa} \alpha}{\sqrt{\alpha^{2}+1}}<1$. This result means that the laser frequency is continuously tuned during the frequency-shifted feedback under this condition, as is the laser intensity. This situation is called weak feedback. The amplitude and phase of the laser intensity is modulated continuously without any abrupt changes, as illustrated in Fig. 2. Therefore, under very weak feedback, the movement information of the targets loaded on the modulated intensity signals can be demodulated to recover the information.

However, when $\frac{\gamma_{c} \tau \sqrt{\kappa} \alpha}{\sqrt{\alpha^{2}+1}}>1$, Eq. (11) has multiple solutions in the region bounded by two special points, which have a vertical slope on the curve of $\omega$ versus $t$, as reported by Ref 25 . This area is well known as the hysteresis region, which is an unstable region. When the laser frequency is increased to one of these points, the frequency skips the unstable region and suddenly drops down to another stable region in the nearest neighbouring period. Correspondingly, the laser intensity also changes abruptly. Accordingly, representative spiking pulses superpose the modulated laser intensity expressed by Eq.(12), as observed in Fig. 9 (a1) and (b1). 

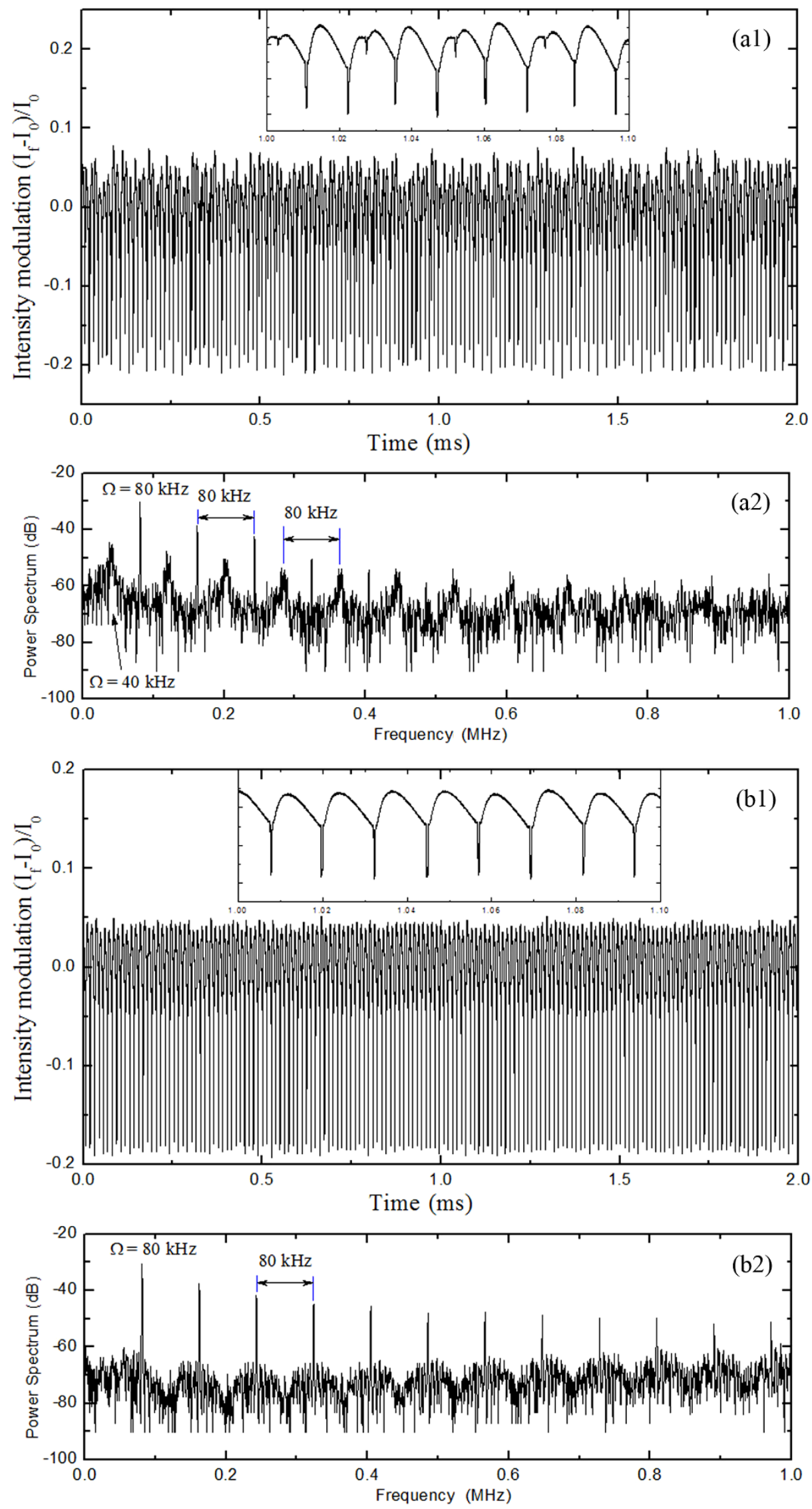

Figure $9 \mid$ Strong response of the laser to external frequency-shifted feedback: $(\mathrm{a}) \boldsymbol{\kappa}=2 \times 10^{-3}(\Omega, \Omega / 2)$ and $(\mathrm{b}) \boldsymbol{\kappa}=2 \times 10^{-3}(\Omega)$. The insert displays a temporal zoom of the intensity. 

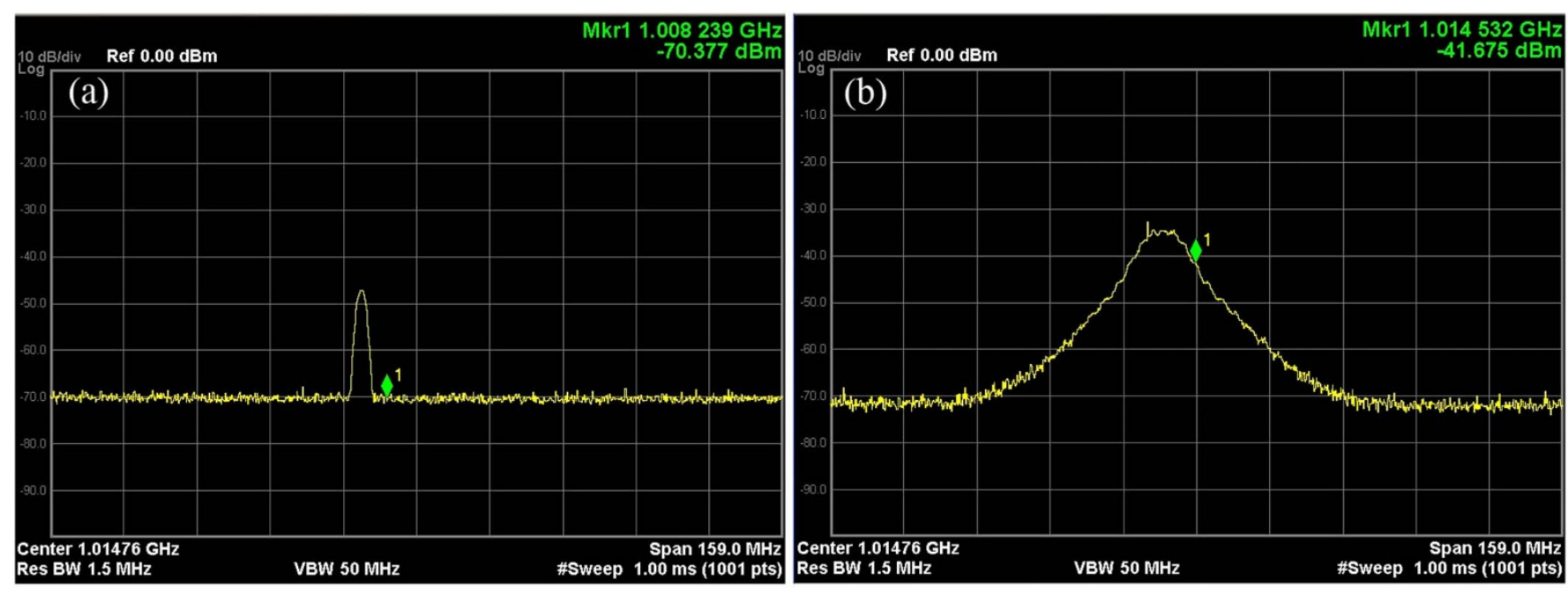

Figure 10 Spectral linewidth broadening of Nd:YAG laser subjected to external frequency-shifted feedback under strong feedback level: (a) without feedback and (b) with feedback $\left(\kappa=2 \times 10^{-3}\right)$.

By straightforwardly solving Eq. (10), the laser intensity $|E|^{2}$ and the corresponding power spectrum can be obtained. Fig. 11 presents the numerical simulation of the intensity and relevant power spectrum for the laser subjected to external frequency-shifted feedback at weak and strong levels, which agree well with the experimental results in Figs. 2 and 9. The values used for numerical simulation are summarised bellow: $\gamma_{c}=6 \times 10^{9} \mathrm{~s}^{-1}, \gamma_{c}=4 \times 10^{3} \mathrm{~s}^{-1}, \alpha=2, \tau=$ $10^{-8} \mathrm{~s}$, all of which are related to the parameters of the laser resonator and external cavity. Other parameters, such as Einstein coefficient B and the pumping rate $\gamma_{1} \mathrm{~N}_{0}$ can be simplified in the arithmetic process. Details about solving the differential equations (10) can be found in Ref 18.

In a similar manner to laser diodes ${ }^{27}$, the linewidth broadening of microchip solid-state lasers in the presence of frequency-shifted feedback can be defined as:

$$
\Delta v=\frac{\Delta v_{0}}{\left[1+C \cos \left(2 \pi \Omega t-\omega \tau+\varphi_{\alpha}\right)\right]^{2}}
$$

where $C=\frac{\gamma_{c} \tau \sqrt{\kappa} \alpha}{\sqrt{\alpha^{2}+1}}, \Delta v$ is the broadened laser spectral linewidth, and $\Delta v_{0}$ is the solitary laser spectral linewidth. According to Eq.(13), the broadened spectral linewidth is related to $\alpha$, feedback level $\kappa$, and the roundtrip time in the external cavity. Under the weak feedback level $\left(\kappa<10^{-4}\right)$, there is no evident broadening in the laser linewidth. However, it is clear that the linewidth can be broadened considerably for a large value of $\mathrm{C}$. Although large narrowing of the laser linewidth by three orders of magnitude has been observed in laser diodes ${ }^{28}$, we did not observe any significant linewidth narrowing in our experiments. The linewidth broadening with a maximum $\Delta v / \Delta v_{0} \sim 10$
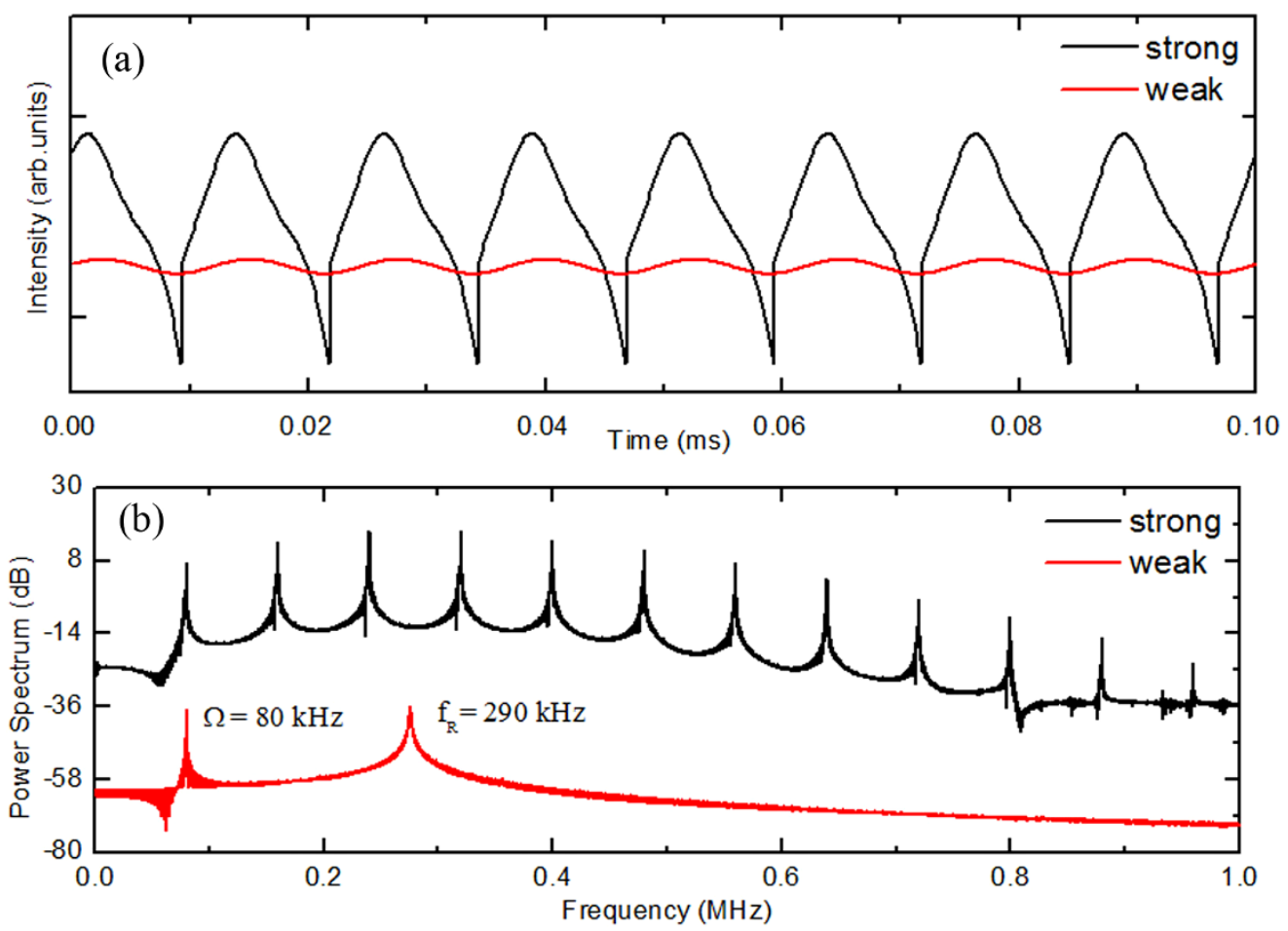

Figure $11 \mid$ Numerical simulation of the response at $\kappa=10^{-6}$ and $\kappa=2 \times 10^{-3}$ : (a) laser intensity and (b) power spectrum. 
occurred when chaotic spiking oscillation at the shifting frequency appeared, as demonstrated in Fig. 10. Along with the increase of the feedback level or the shifting frequency, larger broadening of laser spectral linewidth can be further achieved.

In conclusion, we investigate the weak and strong response of lasers to external frequency-shifted feedback. Based on a weak response, a contact-free measurement scheme combining external frequency-shifted feedback and frequency multiplexing has been developed to achieve high sensitivity, high accuracy and high environmental robustness. Its application in the measurement of displacement, vibration, liquid evaporation, and thermal expansion has also been experimentally demonstrated. Furthermore, spectral linewidth broadening of the order of 10 is observed in the condition of strong response regime. A theoretical analysis that agrees well with the experiments is also presented.

\section{Methods}

Differential frequency-shifting. A pair of AOMs is used in the differential frequency-shifting model. The laser beam (frequency $\omega$ ) passes through the $\mathrm{AOM}_{1}$ (driven at $\Omega_{1}$ ), whose output contains 0 -order (frequency $\omega$ ) and -1-order (frequency $\left.\omega-\Omega_{1}\right)$ diffracted beams. The distance between the two AOMs is kept short enough to ensure that both the output from $\mathrm{AOM}_{1}$ pass through the aperture of $\mathrm{AOM}_{2}$ (driven at $\Omega_{2}$ ). Simultaneously, the incident angle of the laser beam at $\mathrm{AOM}_{2}$ is carefully adjusted to ensure the generation of a +1 -order diffracted beam. Then, after $\mathrm{AOM}_{2}$, four laser beams are obtained, whose frequencies are $\omega, \omega-\Omega_{1}, \omega+\Omega_{2}$, and $\omega-\left(\Omega_{1}\right.$ $\left.-\Omega_{2}\right)$. Among these beams, the beams whose frequencies are $\omega$ and $\omega-\left(\Omega_{1}-\Omega_{2}\right)$ are selected as reference and measurement arms, which return to resonator along the incoming path of the beam (frequency $\omega-\left(\Omega_{1}-\Omega_{2}\right)$ ). Thus, this process is called differential frequency-shifting. The -1-order diffracted beams of $\mathrm{AOM}_{1}$ and the +1order diffracted beams of $\mathrm{AOM}_{2}$ are combined to obtain the differential effect. The relative position of the AOMs to each other is important in constructing this differential frequency-shifting.

The gain amplification $\mathrm{G}(\Omega)$ is a frequency-dependent factor. The closer the $\Omega$ approaches to $f_{R}$, the larger the $G(\Omega)$ is. Thus, in the practical laser feedback interferometer, to get larger gain amplification $\mathrm{G}(\Omega)$ and thereby to sense the measured target with extremely low reflectivity, the frequency shifting of $\Omega$ (typical value $200 \mathrm{kHz})$ is more close to the $\mathrm{f}_{\mathrm{R}}(290 \mathrm{kHz})$ than that $(80 \mathrm{kHz})$ in the experiments.

Signal processing and data analysis. Both the measurement and reference signals are filtered and amplified and are then sent to a dual-channel phase meter (Pretech Science, PT-1313B-2D, China) for simultaneous demodulation to obtain the movement information of the measured targets and the environmental disturbance. Afterwards, the data are transmitted to a computer by USB. A graphic interface programmed by Labview software (developed by National Instruments, USA) is used to read the data, to analyse the data, and to display the final results.

1. Masahiko, F., Keiichi, K. \& Roy, L. Low-frequency intensity fluctuation in laser diode with external optical feedback. Appl. Phys. Lett. 38, 217-220 (1981).

2. Viktorov, E. \& Mandel, A. P. Low frequency fluctuations in a multimode semiconductor laser with optical feedback. Phys. Rev. Lett. 85, 3157-3160 (2000).

3. Agrawal, G. P. Effect of fiber-far end reflections on intensity and phase noise in GaAsp semiconductor laser. Appl. Phys. Lett. 45, 957-959 (1984).

4. Yasaka, H., Yoshikuni, Y. \& Kawaguchi, H. FM noise and spectral line width reduction by incoherent optical negative feedback. IEEE J. Q. E. 27, 193-204 (1991).

5. Petermann, K. Laser Diode Modulation and Noise. 251-256 (Springer, Netherlands, 1991).

6. Sacher, J., Elsasser, W. \& Gobel, E. Intermittency in the coherence collapse of a semiconductor laser with external feedback. Phys. Rev. Lett. 63, 2224-2227 (1989).

7. Li, H., Ye, J. \& Mcinerney, J. G. Detailed analysis of coherence collapse in semiconductor lasers. IEEE J. Q. E. 33, 2421-2431 (1993).

8. Tromborg, B. \& Mork, J. Nonlinear injection locking dynamics and the onset of coherence collapse in external cavity laser. IEEE J. Q. E. 26, 642-654 (1990).

9. Cheng, D. L., Yen, T. C., Chang, J. W. \& Tsai, J. K. Generation of high-speed singlewavelength optical pulses in semiconductor lasers with orthogonal-polarization optical feedback. Opt. Commun. 222, 363-369 (2003).

10. Sciamanna, M. et al. Optical feedback induces polarization mode hopping in vertical-cavity surface-emitting lasers. Opt. Lett. 28, 1543-1545 (2003).
11. Panajotov, K. et al. Polarization switching induced by phase change in extremely short external cavity vertical-cavity surface-emitting lasers. Appl. Phys. Lett. 84, 2763-2765 (2004).

12. Lacot, E., Day, R. \& Stoecke, F. Laser optical feedback tomography. Opt. Lett. 24, 744-746 (1999)

13. Xu, C. X., Tan, Y. D., Zhang, S. L. \& Zhao, S. J. The structure measurement of micro-electro-mechanical system devices by the optical feedback tomography technology. Appl. Phys. Lett. 102, 221902 (2013).

14. Wong, T. L., Sabato, S. L. \& Bearden, A. A prototype scanning laser-feedback microscope for imaging biological cells in aqueous media. Journal of Microscopy 177, 162-170 (1995).

15. Wan, X. J., Li, D. \& Zhang, S. L. Quasi-common-path laser feedback interferometry based on frequency shifting and multiplexing. Opt. Lett. 32, 367-369 (2007)

16. Otsuka, K., Abe, K., Ko, J. Y. \& Lim, T. S. Real-time nanometer-vibration measurement with a self-mixing microchip solid-state laser. Opt. Lett. 27, 1339-1341 (2002).

17. Kawai, R., Asakawa, Y. \& Otsuka, K. Ultrahigh-sensitivity self-mixing laser Doppler velocimetry with laser-diode-pumped microchip $\mathrm{LiNdP}_{4} \mathrm{O}_{12}$ lasers. IEEE. Photonic. Tech. Lett. 11, 706-708 (1999).

18. Tan, Y. D., Xu, C. X. \& Zhang, S. L. Power spectral characteristic of microchip Nd:YAG laser subjected to frequency-shifted optical feedback. Laser Phys. Lett. 10, 025001 (2013).

19. Reverter, F., Li, X. J. \& Meijer, G. C. M. Liquid-level measurement system based on a remote grounded capacitive sensor. Sensor. Actuat. A-Phys. 138, 1-8 (2007).

20. Ilev, I. K. \& Waynant, R. W. All-fiber-optic sensor for liquid level measurement. Rev. Sci. Instrum. 70, 2551-2554 (1999).

21. Chakravarthy, S., Sharma, R. \& Kasturi, R. Noncontact level sensing technique using computer vision. IEEE T. Instrum. Meas. 51, 353-361 (2002).

22. Steward, E. G. \& Cook, B. P. X-ray measurement of thermal expansion perpendicular to the layer planes of artificial and natural graphites. Nature $\mathbf{1 8 5}$, 78-80 (1960).

23. Ye, J. P. et al. Bidirectional thermal expansion measurement for evaluating Poisson's ratio of thin films. Appl. Phys. Lett. 89, 031913 (2006).

24. Giuliani, G. \& Donati, S. Laser interferometry in Unlocking Dynamical Diversity: Optical Feedback Effects on Semiconductor Lasers. Kane, D. M. (ed.) \& Shore, K. A. (ed.), 220-229 (Wiley, England, 2005).

25. Acket, G. A., Lenstra, D., Den Boef, A. \& Verbeek, B. The influence of feedback intensity on longitudinal mode properties and optical noise in index-guided semiconductor lasers. IEEE J. Q. E. 20, 1163-1169 (1984).

26. Lang, R. \& Kobayashi, K. External optical feedback effects on semiconductor injection laser properties. IEEE J. Q. E. 16, 347-355 (1980).

27. Agrawal, G. P. Line narrowing in a single-mode injection laser due to external optical feedback. IEEE J. Q. E. 20, 468-471 (1984).

28. Wyatt, R. \& Delin, W. J. $10 \mathrm{kHz}$ linewidth $1.5 \mu \mathrm{m}$ InGaAsP external cavity laser with $55 \mathrm{~nm}$ tuning range. Electron. Lett. 19, 110-112 (1983).

\section{Acknowledgments}

This work was financially supported by the National Natural Science Foundation of China (Grant No. 50805084), Tsinghua University Initiative Scientific Research Programme (2012Z02166) and the Special-funded Programme on National Key Scientific Instruments and Equipment Development of China (2011YQ04013603)

\section{Author contributions}

Y.T. designed the experiments, developed the concept, constructed the optical hardware and wrote this paper. S.Z. designed the experiments, developed the concept and supervised the entire project. S.Z. implemented the experiments of liquid evaporation. Y.Z. performed the heat expansion experiments. N.L. was responsible for the data acquisition and software.

\section{Additional information}

Competing financial interests: The authors declare no competing financial interests.

How to cite this article: Tan, Y., Zhang, S., Zhang, S., Zhang, Y. \& Liu, N. Response of microchip solid-state laser to external frequency-shifted feedback and its applications. Sci. Rep. 3, 2912; DOI:10.1038/srep02912 (2013)

This work is licensed under a Creative Commons AttributionNonCommercial-NoDerivs 3.0 Unported license. To view a copy of this license, visit http://creativecommons.org/licenses/by-nc-nd/3.0 